\title{
Exploring nanomagnetism with soft $X$-ray microscopy
}

Peter Fischer, Dong-Hyun Kim, Brooke L. Mesler, Weilun Chao, Anne E. Sakdinawat, Erik H. Anderson

Center for X-ray Optics

Lawrence Berkeley National Laboratory

Berkeley CA U.S.A.

PJFischer@lbl.gov

http://www.cxro.lbl.gov/BL612

\section{Corresponding author:}

Peter Fischer

Center for X-ray Optics

Lawrence Berkeley National Laboratory

MS 2-400

1 Cyclotron Road

Berkeley CA 94720

U.S.A.

phone : +1 5104867052

fax: +15104864550

email: PJFischer@lbl.gov

http://www.cxro.lbl.gov/ pjfischer/index.php 


\begin{abstract}
Magnetic soft X-ray microscopy images magnetism in nanoscale systems with a spatial resolution down to $15 \mathrm{~nm}$ provided by state-of-the-art Fresnel zone plate optics. X-ray magnetic circular dichroism (X-MCD) is used as element-specific magnetic contrast mechanism similar to photoemission electon microscopy (PEEM), however, with volume sensitivity and the ability to record the images in varying applied magnetic fields which allows to study magnetization reversal processes at fundamental length scales. Utilizing a stroboscopic pump-probe scheme one can investigate fast spin dynamics with a time resolution down to $70 \mathrm{ps}$ which gives access to precessional and relaxation phenomena as well as spin torque driven domain wall dynamics in nanoscale systems. Current developments in zone plate optics aim for a spatial resolution towards $10 \mathrm{~nm}$ and at next generation X-ray sources a time resolution in the fsec regime can be envisioned.
\end{abstract}

\title{
Keywords
}

magnetic soft X-ray microscopy, X-ray optics, Fresnel zone plates, magnetic nanostructures, magnetization reversal, fast spin dynamics, X-ray magnetic circular dichroism 


\section{Introduction}

Magnetism on the nanometer scale and its spin dynamics on a nsec to fsec time scale is currently a scientfically highly attractive topic $[1,2]$ since it addresses both fundamental magnetic lengh scales such as magnetic exchange lengths in the sub-10nm range [3] and interesting time scales of magnetism such as precessional and relaxation phenomena, domain wall and vortex dynamics [4-6]. The fundamental time scale in magnetism is given by the time required to transfer energy and momentum from the electronic into the spin system [7], which aims e.g. to understand the time scale on which magnetization can evolve upon excitation e.g. with ultrafast optical pulses $[8,9]$. There is also a strong technological interest in fundamental magnetization processes on the nanometer length and nsec time scale due to current developments in ultrahigh density magnetic data storage media and miniaturized magnetic sensor technology. It is still an open question how to control e.g. the switching field distribution in future magnetic storage devices where the bit size approaches the granular length scale. New technological concepts such as spintronics, where in addition to the charge the spin of the electron is considered, require to control the electron spin on a nanoscale with ps timing.

Recently it has been shown that apart from switching the magnetization with a magnetic (Oersted) field, the concept of creating a spin torque that acts onto the local magnetization by injecting spin polarized currents into a magnetic device is a revolutionary concept to switch the magnetization on the nanoscale [10-15]. Logical elements for spintronics, non-volatile magnetic random access memories (MRAM) or 3dim magnetic data storage devices are just a few of the potential applications [16]. From the materials science point of view the quest is open to find novel and smart materials that will provide particular functionalities. Typical examples are e.g 
multiferroic systems which hold the promise to switch the magnetization e.g. with electrical fields [17-20].

New analytical tools are required and a direct visualization of the magnetization is very appealing. The grand challenge to modern magnetic microscopies is therefore to provide both a spatial resolution in the nanometer regime, a time resolution on a ps to fs scale and elemental specificity which allows to study such advanced magnetic materials. Magnetic transmission soft X-ray microscopy (MTXM) is a powerful novel technique that combines X-ray magnetic circular dichroism (X-MCD) as huge and element specific magnetic contrast mechanism with high spatial and temporal resolution down to $15 \mathrm{~nm}$ and $70 \mathrm{ps}$, resp.[21-25]. As a pure photon-in/photon-out based technique the images can be recorded in external magnetic fields giving access to study magnetization reversal phenomena on the nanoscale.

This paper reviews the current status of magnetic soft X-ray microscopy with regard to spatial and temporal resolution. It is complementary to X-PEEM regarding its bulk (volume) sensitivity compared to surface sensitivity in X-PEEM. This allows soft X-ray microscopy to access the nanomagnetism e.g. in multilayered systems with a thickness much larger than the electron escape depth. Typical examples will demonstrate the achievements in spatial resolution and time resolved X-ray microscopy. Recent developments of novel X-ray optics allow for magnetic phase contrast imaging, which can be of importance at high brillant upcoming X-ray sources that will give access to fsec and single shot spin dynamics with less than 10nm spatial resolution.

\section{Experimental Details}

There are three basic requirements to perform magnetic soft X-ray microscopy with high spatial and temporal resolution, namely high resolution X-ray optics, circular polarization of soft X-rays and a fast time structure of the X-ray source 
Fresnel zone plates (FZPs) are the key compoents to soft X-ray microscopy. Due to the refractive index of soft X-rays, i.e. photons with energies between $200-2000 \mathrm{eV}$, being close to unity, conventional lenses cannot be used. State-of-the-art FZPs used as X-ray optical elements in soft X-ray microscopy provide a spatial resolution down to currently $<15 \mathrm{~nm}[24,26]$. FZPs are circular diffration gratings with a radially increasing line density, i.e they consist of alternating transparent and opaque rings. They can be fabricated using state-of-the-art nanolithography. The three parameters defining the performance of a FZP is the outermost zone width $(\Delta r)$, the number of zones $(N)$ and the wavelength $(\lambda)$ [27]. From these three parameters all other ZP parameters can be derived such as focal length $(f)$, diameter $(D)$, numerial aperture $(N A)$. The spatial resolution of a zone plate based microscope is equal to $k_{1} \lambda / N A_{M Z P}$, where $k_{1}$ is an illumination dependent constant, which ranges from 0.3 to 0.61 . For a partially coherent illumination $k_{l}$ has a typical value of 0.4 and with $N A_{M Z P}=\lambda / 2 \Delta r$ for high magnification the theoretical resolution for a full field microscope, as described here is $0.8 \Delta r[26]$.

Soft X-rays are abundantly available at synchrotron radiation sources. Moreover they provide circularly polarized X-rays which are required to use XMCD as magnetic contrast mechanism. For bending magnet sources the radiation viewed at an inclined angle achieves elliptical polarization with reversed helicity above and below the orbital plane which can be selected readily by appropriated apertures [28]. Dedicated helical undulator sources provide means to tune the circular polarization by mechanical moving the magnet array structures in the undulator source and switching the helicity can be achieved within few seconds only. Synchrotron radiation also exhibits an inherent time structure which is determined (and currently limited) by the lengths of the electron bunches circulating in the storage ring 
at typically $500 \mathrm{MHz}$. Thus fast magnetization dynamics with typically $<100 \mathrm{ps}$ time resolution can be performed by applying stroboscopic pump-probe schemes.

Fig. 1 shows the schematics of XM-1, the high resolution soft X-ray microscope located at beamline 6.1.2 at the Advanced Light Source in Berkeley CA (USA), which is currently the only system worldwide which is dedicated to studies of nanomagnetism [21]. It has been built in 1994 by the Center for X-ray Optics (CXRO) and has been used since then for a large variety of applications, ranging from nanomagnetism, materials and environmental science to biological applications by a large number of users. It exhibits a user-friendly design so as to allow to record several thousands of Xray images per day.

The principle of the soft X-ray microscope XM-1 for magnetic imaging is described elsewhere [23]. Similar to an optical microscope XM-1 consists of two optical components, namely a condenser and an objective lense, however these comprise FZPs. First, a condensor zone plate together with a pinhole close to the sample forms a linear monochromator due to the wavelength dependence of the focal length of FZPs and second, the radiation passing through the sample is projected through the micro zone plate (MZP), which is the lens determining the lateral resolution, onto a CCD camera, where the images are recorded (see Fig. 1 [21]).

To image magnetic nanostructures the off-orbit emitted circularly polarized X-rays are selected by a movable aperture system upstream the CZP. The spectral resolution that can be obtained at XM-1 with a typical value of $\Delta E / E=500$ is sufficient to discriminate the spin-orbit separated $\mathrm{L}_{3,2}$ edges $(\sim 10-20 \mathrm{eV})$ in transition metals such as $\mathrm{Fe}, \mathrm{Co}, \mathrm{Ni}$ occuring at around $700-900 \mathrm{eV}$, so that $\mathrm{XMCD}$ can be used as magnetic contrast mechanism. 
As a pure photon-in/photon-out based microscopy technique external magnetic field can be applied during the recording so as to investigate magnetization reversal processes, local switching field distributions etc. Typical exposure times for magnetic imaging range from $0.5-3 \mathrm{sec}$ typically and each image covers about $10-15 \mu \mathrm{m}$ field-of-view. To eliminate non-magnetic background structures in the raw data, one can either normalize to a magnetically fully saturated image or reverse the magnetic structures by taking two images with reversed helicity. Studies of spin dynamics on the nanoscale combines the high spatial resolution of magnetic soft X-ray microscopy with a stroboscopic pump-probe scheme utilizing the inherent time structure of the emitted X-ray pulses [29]. At the ALS there are two modes of operating the storage ring. In multibunch mode the electrons with a typical energy of $1.9 \mathrm{GeV}$ circulate at a frequency of $499.642 \mathrm{MHz}$ in 276 bunches with a length of each 70ps and a distance between neighboring bunches of $2 \mathrm{~ns}$ thus providing a total electron current of $400 \mathrm{~mA}$. With a $196.8 \mathrm{~m}$ circumference of the ring there is a gap in the storage pattern, which is filled with a single (camshaft) bunch of $10 \mathrm{~mA}$. The second mode of operation is the two-bunch mode where there are two electron bunches stored each of them with $20 \mathrm{~mA}$ current and 70ps lengths and separated by $328 \mathrm{~ns}$, i.e. emitting 3MHz X-ray flashes that can be used for time resolved spin dynamics experiments at XM-1.

\section{Results}

$\mathrm{X}$-ray magnetic circular dichroism detects the difference in the magnetic photoabsorption cross section which depends on the relative orientation between the projection of the speciimen's magnetization onto the photon propagation direction and the helicity of the transmitting X-rays. Strong X-MCD effects up to tens of percent occur in the vicinity of element-specific $\mathrm{L}_{3}$ and $\mathrm{L}_{2}$ absorption edges of transition metals 
e.g. $\mathrm{Fe}, \mathrm{Co}, \mathrm{Ni}$ which correspond to the binding energies of inner core atomic levels, such as the $2 p_{3 / 2}$ and $2 p_{1 / 2}$ levels, resp.. Since the magnetic ground state of a ferromagnetic system in general splits up into a magnetic domain configuration [30], i.e. in order to minimize competing magnetic energies, such as anisotropy, exchange, dipolar and Zeeman energy it is more favorable to create neighboring areas where the magnetization points in opposite directions, the transmission of circularly polarized soft X-rays will differ locally which can be visualized by imaging the transmitted X-rays with sufficient spatial resolution. Since magnetic thin films exhibit in general an inplane magnetization to avoid magnetic charges, these sample have to be tilted with respect to the incoming photon beam to record the projection of the magnetization. A typical example of high resolution magnetic imaging with soft X-ray microscopy is shown in Fig. 2 [24]. The magnetic domain structure of a $50 \mathrm{~nm}$ thin nanogranular $\left(\mathrm{Co}_{83} \mathrm{Cr}_{17}\right)_{87} \mathrm{Pt}_{13}$ alloy film with a pronounced perpendicular magnetic anisotropy and its nucleation and magnetization reversal behaviour in external applied magnetic fields was recorded at the $\mathrm{Co}_{3}$ absorption edge. The dark and white areas in the image correspond to the direction of the Co magnetic moments. TEM analysis of this film revealed a grain size distribution with an average grain size of about $20 \mathrm{~nm}$. From the intensity profile shown in the bottom panel of Fig. 2 a $15 \mathrm{~nm}$ resolution, provided by the high resolution MZP was obtained, which indicates that these results provide insight into the switching of individual grains in that system which is discussed as candidate for perpendicular recording.

Apart from imaging the static magnetism at fundamental magnetic length scales, there is an increasing interest to understand also fast spin dynamics occuring on the nanoscale [31-33]. However, since the number of photons per X-ray pulse at current facilities is not sufficient to create single-shot images, a stroboscopic pump-and-probe measurement is used (see Fig. 3) to record the magnetization dynamics in nanoscale 
magnetic elements with time resolved magnetic X-ray microscopy. As a pump pulse a short electronic current pulse is used with a rise time of less than 100ps and a typical pulse length of up to several 1ns. When launched into e.g. planar waveguide structures one can generate subsequent short and instantaneous magnetic field pulses, which excite various modes of the local spin configuration. There is increased interest to excite not only with classical Oersted fields, but moreover to inject spin polarized currents into nanoscale materials and thus create a spin torque e.g. on the domain walls. Such dynamics can also be addressed by time resolved X-ray microscopy.

The probe is the X-ray flash of the synchrotron which can be delayed with respect to the pump pulse up to several ns in steps of several ps to study the temporal evolution of the magnetization after the exciting pulse. Typical accumulation times per single image are about 2-5 sec and about 25-50 images are summed for each time step. As with the static imaging non-magnetic background contributions can be suppreseed by normalizing to a magnetically fully saturated image obtained by applying a strong external magnetic field up to few kOe field strength.

A typical example where the vortex dynamics in a rectangular permalloy $\left(\mathrm{Fe}_{19} \mathrm{Ni}_{81}\right)$ microelement has been studied is shown in Fig. 4. The observed seven domain Landau pattern and its associated spin configuration is displayed in Fig. 4 (a) together with the direction of the field pulse and the external magnetic field. Fig. 4 (b) and (c) show two X-ray images obtained at different delay times between the pump and the probe. One can clearly see that upon exciation the vortices in the Landau pattern have been displaced. Following the full sequence of the dynamics, one sees the precessional motion of the two vortices with a cycling time of several nsec.

\section{Conclusion}


Magnetic soft X-ray microscopy has achieved a spatial resolution of $15 \mathrm{~nm}$ which is close to fundamental magnetic length scales. Magnetic nanostructures, domain walls, media at the grain size level can be imaged and its reversal behaviour in external magnetic fields. The inherent elemental sensitivity due to XMCD as magnetic contrast mechanism is valuable in view to multilayered, multicomponent novel and advanced magnetic materials, such as magnetic multilayersm, diluted magnetic semiconductors and multferroic systems, which combine different order parameters and hold great promise as novel technological relevant nanoscale (quantum) materials with outstanding novel functionalities.

So far, time resolved X-ray microscopy is limited in two senses. Firstly, only reproducible processes can be studied due to the limited number of photons available at current X-ray sources. Secondly, the current time resolution of soft X-rays is far away from fundamental time scales that are associated with the time scale of exchange interaction and magnetization fluctuations in the fsec regime. However, upcoming Xray free electron lasers and other high brightness coherent and incoherent sources for soft X-ray offer the opportunity for single shot imaging of fs spin dynamics with soft X-ray microscopy. Even more, the continous development of suited X-ray optics is crucial. To minimize the photon load onto the sample, an interesting opportunity is to use magnetic phase contrast imaging. Recently, it has been shown that with Fourier optical elements, a magnetic differential interference contrast could be obtained [34]. An XOR zone plate, which was used instead of the conventional MZP demonstrated magnetic phase contrast showing up at a photon energy below the absorption edge, where the flux of photons can be reduced significantly. Fig. 5 (a) shows the scheme of the zone plate optics and the results obtained around the $\mathrm{L}_{3,2}$ absorption edge of a $\mathrm{Gd}_{25} \mathrm{Fe}_{75}$ amorphous film are displayed in Fig $5(\mathrm{~b}, \mathrm{c})$. The boundary of the magnetic domain patterns are enhanced at a photon energy of $705 \mathrm{eV}$ and $711 \mathrm{eV}$, where no 
absorption contrast is expected. The observed reversal of contrast is in agreement with measurements of the magnetic real part of the refractive index [35].

It can be foreseen, that magnetic soft X-ray microscopy will play an important role in the future to study spin structures on the nanoscale with fsec time resolution.

\section{Acknowledgements}

The valuable help of the technical staff of CXRO and the ALS, particularly R. Oort,

S. Rekawa, P. Denham, J: Gamsby, B. Gunion, K. Bradley, D. Kemp, and R. Tackaberry is highly appreciated. We highly appreciate the excellent collaboration with C. Chang (U Drexel) on magnetic phase contrast imaging and M.-Y. Im, S.-C. Shin (KAIST Korea) on the nanogranular media. We would like to express our thanks to D. Attwood (CXRO) for his continued support of our research.

This work was supported by the Director, Office of Science, Office of Basic Energy

Sciences, Materials Sciences and Engineering Division, of the U.S. Department of Energy under Contract No DE-AC02-05-CH11231.

\section{References}

[1] J. Miltat, A. Thiaville, Science 290, (2000) 466.

[2] M.R. Freeman, B.C. Choi, Science 294, (2001) 1484.

[3] A. Wachowiak, J. Wiebe, M. Bode, O. Pietzsch, M. Morgenstern, R. Wiesendanger, Science 298 (2002) 577

[4] S.-B. Choe, Y. Acremann, A. Scholl, A. Bauer, A. Doran, J. Stohr, and H.A. Padmore, Science 304, (2004), p 420

[5] Y. Acremann et al., Science 290, (2000) 492.

[6] W.K. Hiebert, G.E. Ballentine, L. Lagae, R.W. Hunt, M.R. Freeman, J. Appl. Phys. 92(1) (2002) 392 
[7] J. Stoehr and H.C. Siegmann, Magnetism, Springer (2006)

[8] T. Gerrits, H.A.M. van den Berg, J. Hohlfeld, L. Bär, T. Rasing, Nature 418 (2002) 511.

[9] V. Kimel, et al, Nature 435 (2005) 655

[10] Y. Acremann et al, Phys Rev Lett, 96 (2006) 217202

[11] J.Z. Sun, IBM J. Res. Dev. 50(1) 2006, 81

[12] M. Hayashi, et al., Phys. Rev. Lett. 96 (2006) 197207

[13] L. Thomas et al., Nature, 443, (2006) 197

[14] J. C. Slonczewski, J. Magn. Magn. Mater. 159, (1996) L1

[15] L. Berger, J. Appl. Phys. 55 (1984) 1954

[13] S. S. P. Parkin, U.S. Patent No. 6834005 (2004)

[14] M. Kläui et al, Appl. Phys Lett., 88 (2006) 232507

[15] S. Mangin, et al., Nature Mat. 5 (2006) 210

[16] D.A. Allwood, et al., Science 309 (2005)1688

[17] T : Kimura et al, Nature 426 (2003) 55

[18] A : Pimenov, et al., Nature Phys. 2 (2006) 97

[19] J. Wang et al., Science, 299, (2003) 1719

[20] M .Fiebig, J. Phys. D : Appl Phys. 38 (2005) R123

[21] P. Fischer, D.-H. Kim, W. Chao, J.A. Liddle, E.H. Anderson, and D.T. Attwood, Materials Today 9(1-2) (2006) 26

[22] P. Fischer, et al, Z. f. Physik B 101 (1996) 313

[23] P. Fischer et al., Rev. Sci. Instr. 72(5) (2001) 2322-24

[24] D.-H. Kim, P. Fischer, W. Chao, E.Anderson, M.-Y. Im, S.-C. Shin, and S.-B. Choe, J. Appl. Phys. 99 (2006) 08H303 
[25] H. Stoll, A. Puzic, B. van Waeyenberge, P. Fischer, J. Raabe, M. Buess, T. Haug, R. Hoellinger, C.Back, D. Weiss, and G. Denbeaux, Appl. Phys. Lett. 84(17) (2004) 3328

[26] W. Chao, B.D. Harteneck, J.A. Liddle, E.H. Anderson, and D.T. Attwood, Nature 435 (2005) 1210

[27] D.T. Attwood, Soft X-rays and Extreme Ultraviolet Radiation: Principles and Applications (Cambridge University Press, 1999)

[28] B.S. Kang, D.H. Kim, E. Anderson, P. Fischer, G. Cho, J. Appl. Phys. 98 (2005) 093907

[29] P. Fischer et al, J. Phys IV France 104 (2003) 471

[30] A. Hubert and R. Schäfer, Magnetic Domains, Springer, Berlin, (1998)

[31] A. Himeno, S. Kasai, and T. Ono, Appl. Phys. Lett. 87, (2005) 243108

[32] T. Shinjo, T. Okuno, R. Hassdorf, K. Shigeto, and T. Ono, Science 289 (2000) 930

[33] K.S. Buchanan, P.E. Roy, M. Grimsditch, F.Y. Fradini, K.Y. Guslienko, S.D.

Bader and V. Novosad, Nature Physics 1, (2005) 172

[34] C. Chang, A. Sakdinawat, P. Fischer, E. Anderson, D. Attwood, Optics Letters 31(10) (2006) 1564

[35] J.B: Kortright and S.-K. Kim, Phys Rev B, 62 (2000) 12216 


\section{Figure captions}

Fig. 1 Top: Bird's eye view of the soft X-ray microscope beamline 6.1.2 (XM-1)

located at the Advanced Light Source in Berkley, CA. The X-rays enter from the left through the concrete wall into the microscope.

Bottom: Schematics of the X-ray optical layout of XM-1 [21].

Fig. 2 Top: Magnetic X-ray image of the domain structure in a CoCrPt nanocrystalline thin film imaged at the Co L3 absorption edge (777eV) with $15 \mathrm{~nm}$ MZP.

Bottom: Intensity scan across the white bar in the X-ray image (above), indicating a spatial resolution of $<15 \mathrm{~nm}[24]$.

Fig. 3 Schematics of the stroboscopic pump-probe experiment at XM-1 to image time resolved spin dynamics in magneto nanoelements. The pump is an electronic pulse launched into a waveguide structure underneath the magnetic element to create a short magnetic field pulse (rise time 100ps) onto the speciment. The probe pulse are X-ray flashes at $3 \mathrm{MHz}$ with a length of $70 \mathrm{ps}$. To study the dynamics the pump pulse is delayed relattive to the probe pulse up to several tens of nsec.

Fig. 4: Typical example of vortex dynamics in a PY nanoelement exhibiting a seven domain Lanaud configuration. (a) Schematic diagram showing the configuration in the element. The arrows indicate the direction of the magnetization in the film plane. (b) Ground state configuration, i.e. imaged before the pump pulse. (c) Displacement of the vortex structure after the excitation.

Fig. 5: (a) XOR Fourier optical element that replaces the conventional MZP to detext magnetic phase contrast. (b) Magnetic domain structure in an amorphous GdFe thin 
film recorded at $705 \mathrm{eV}$ (below the absorption edge). (c) Magnetic domain structure recorded at $711 \mathrm{eV}$ indication both a magnetic contrast at the boundary (differential contrast detection) and the reversal of the contrast [34]. 


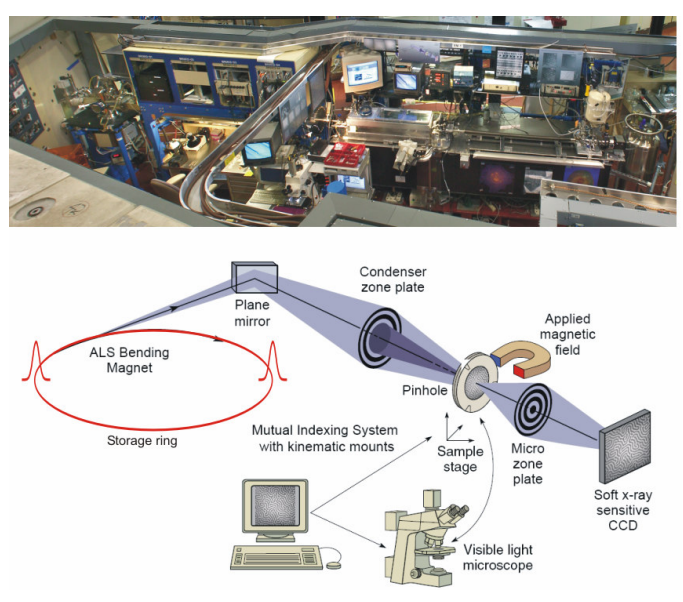

Fig. 1 

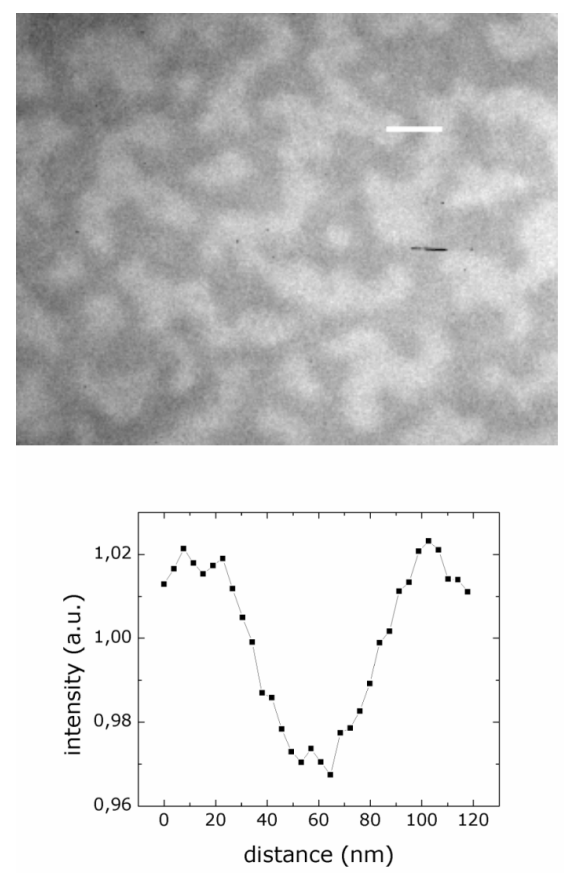

Fig. 2 


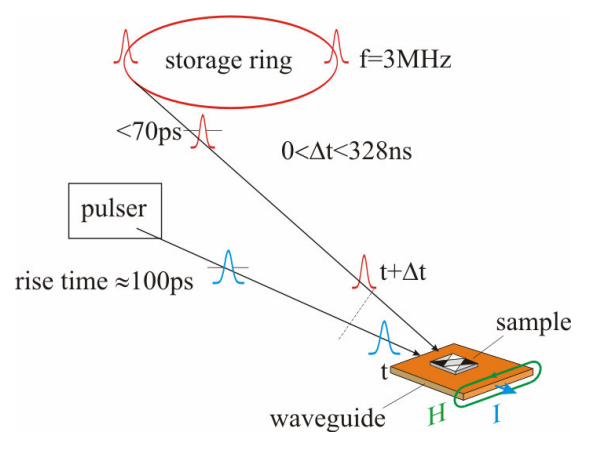

Fig. 3 

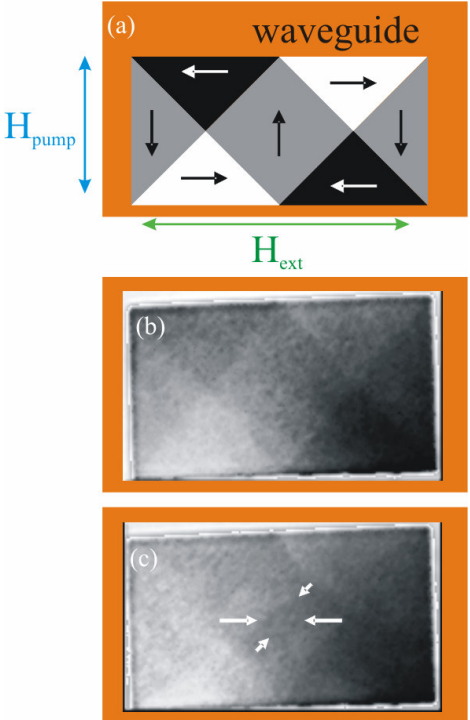

Fig. 4 


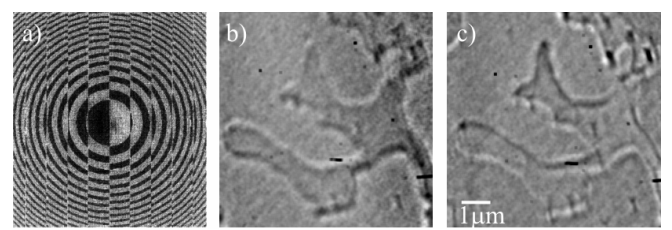

Fig. 5 